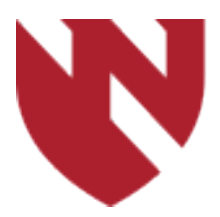

October 2021

\title{
Alcohol Consumption is associated with Increased CEA Levels in Male Patients with Stage IV Colorectal Cancer- A Single-Institution Retrospective Analysis
}

\author{
Lauren M. Keim \\ University of Nebraska Medical Center \\ Alexander W. Praus \\ University of Nebraska Medical Center \\ Walter S. Campbell \\ University of Nebraska Medical Center \\ Kaeli K. Samson \\ University of Nebraska Medical Center \\ Ashley M. Mohr \\ University of Nebraska Medical Center \\ Tell us how you used this information in this short survey.

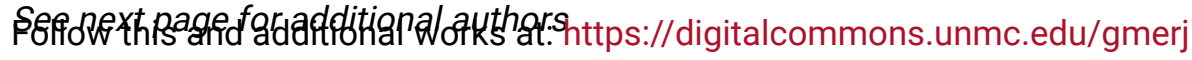 \\ Part of the Neoplasms Commons
}

\section{Recommended Citation}

Keim, L. M., Praus, A. W., Campbell, W. S., Samson, K. K., Mohr, A. M., Tobi, M., , McVicker, B. L. Alcohol Consumption is associated with Increased CEA Levels in Male Patients with Stage IV Colorectal CancerA Single-Institution Retrospective Analysis. Graduate Medical Education Research Journal. 2021 Oct 04; 3(1). https://digitalcommons.unmc.edu/gmerj/vol3/iss1/1

This Original Report is brought to you for free and open access by DigitalCommons@UNMC. It has been accepted for inclusion in Graduate Medical Education Research Journal by an authorized editor of DigitalCommons@UNMC. For more information, please contact digitalcommons@unmc.edu. 


\title{
Alcohol Consumption is associated with Increased CEA Levels in Male Patients with Stage IV Colorectal Cancer- A Single-Institution Retrospective Analysis
}

\begin{abstract}
Introduction: Alcohol use is an independent risk factor for liver metastasis, a major cause of morbidity and mortality in colorectal cancer (CRC) patients. Serum CEA level is an established prognostic indicator in CRC, yet the correlation with behavioral factors such as alcohol use remains to be defined. In a singlecenter review, we evaluated alcohol use, gender, and CEA levels in predicting advanced disease in CRC patients.

Methods: Retrospective analysis of UNMC patients diagnosed with CRC as the primary cancer between 2012-2019, stages I-IV, and age >19 with documentation of alcohol use. Univariable statistics were performed using Chi-Square and non-parametric tests. Associations between stage, gender, and alcohol use (some vs. none) and the log-transformed CEA outcome (either initial or rate of change) were assessed using linear regressions.
\end{abstract}

Results: Alcohol use was found to be reported in 333 of 1243 CRC patients. The cohort was comprised of 192 male and 141 female subjects. Elevated CEA levels at CRC diagnosis were associated with increased all-cause mortality ( $33.0 \%$ for CEA $>3.4 \mathrm{ng} / \mathrm{ml}$ vs $10.4 \%$ for CEA $<3.4 \mathrm{ng} / \mathrm{ml})$. Model analysis found that stage IV male alcohol users showed an increase in serial CEA levels compared to males who did not use alcohol, but this pattern was not observed among stage IV females.

Conclusions: Males with a history of alcohol use may be at risk for advanced CRC disease suggesting the utility of serial serum CEA monitoring in these patients. A detailed alcohol use history should be obtained in all patients with CRC as it has prognostic value and may allow for early intervention. This analysis was limited by missing alcohol use data for the majority (73.2\%) of CRC patients evaluated. A prospective study is warranted to define the implications of alcohol use and risk of CRC liver metastasis.

\section{Keywords}

colorectal carcinoma, alcohol, colorectal liver metastasis, carcinoembryonic antigen

\section{Creative Commons License}

\section{cc) (i) (위}

This work is licensed under a Creative Commons Attribution-Noncommercial-No Derivative Works 4.0 License.

\section{Cover Page Footnote}

The project described utilizes the UNMC Clinical Research Analytics Environment (CRANE). CRANE is supported funding from the National Institute of General Medical Sciences, U54 GM115458 and the Patient Centered Outcomes Research Institute, PCORI CDRN-1306-04631. The content is solely the responsibility of the authors and does not necessarily represent the official views of the NIH or PCORI.

\section{Authors}

Lauren M. Keim, Alexander W. Praus, Walter S. Campbell, Kaeli K. Samson, Ashley M. Mohr, Martin Tobi, and Benita L. McVicker 


\section{Alcohol Consumption is Associated with Increased CEA Levels in Male Patients with Stage IV Colorectal Cancer - A Single-Institution Retrospective Analysis}

Lauren M. Keim ${ }^{1, *}$, Alexander W. Praus ${ }^{1, *}$, Walter S. Campbell ${ }^{2}$, Kaeli K. Samson ${ }^{3}$ Ashley Mohr ${ }^{4}$, Martin Tobi ${ }^{5}$, Benita L. McVicker ${ }^{6}$

${ }^{1}$ University of Nebraska Medical Center, College of Medicine, Department of Internal Medicine

${ }^{2}$ University of Nebraska Medical Center, College of Medicine, Department of Pathology/Microbiology

${ }^{3}$ University of Nebraska Medical Center, College of Public Health, Department of Biostatistics

${ }^{4}$ University of Nebraska Medical Center, College of Medicine, Department of Biochemistry and Molecular Biology

${ }^{5}$ Detroit VAMC and Central Michigan University College of Medicine, Research and Development Service

${ }^{6}$ VA Nebraska-Western lowa Health Care System and the University of Nebraska Medical Center, Research Service and the Department of Internal Medicine

* These authors contributed equally

https://doi.org/10.32873/unmc.dc.gmerj.3.1.001

\begin{abstract}
Introduction: Alcohol use is an independent risk factor for liver metastasis, a major cause of morbidity and mortality in colorectal cancer (CRC) patients. Serum CEA level is an established prognostic indicator in CRC, yet the correlation with behavioral factors such as alcohol use remains to be defined. In a single-center review, we evaluated alcohol use, gender, and CEA levels in predicting advanced disease in CRC patients.
\end{abstract}

Methods: Retrospective analysis of UNMC patients diagnosed with CRC as the primary cancer between 2012-2019, stages I-IV, and age $>19$ with documentation of alcohol use. Univariable statistics were performed using Chi-Square and non-parametric tests. Associations between stage, gender, and alcohol use (some vs. none) and the logtransformed CEA outcome (either initial or rate of change) were assessed using linear regressions.

Results: Alcohol use was found to be reported in 333 of 1243 CRC patients. The cohort was comprised of 192 male and 141 female subjects. Elevated CEA levels at CRC diagnosis were associated with increased all-cause mortality $(33.0 \%$ for CEA $>3.4 \mathrm{ng}$ / $\mathrm{ml}$ vs $10.4 \%$ for CEA $<3.4 \mathrm{ng} / \mathrm{ml})$. Model analysis found that stage IV male alcohol users showed an increase in serial CEA levels compared to males who did not use alcohol, but this pattern was not observed among stage IV females.

Conclusions: Males with a history of alcohol use may be at risk for advanced CRC disease suggesting the utility of serial serum CEA monitoring in these patients. A detailed alcohol use history should be obtained in all patients with CRC as it has prognostic value and may allow for early intervention. This analysis was limited by missing alcohol use data for the majority (73.2\%) of CRC patients evaluated. A prospective study is warranted to define the implications of alcohol use and risk of CRC liver metastasis.

\section{Introduction}

Colorectal cancer (CRC) is the 3rd most common cancer worldwide, and in the U.S. alone, accounts for over 50,000 deaths annually. ${ }^{1} \mathrm{CRC}$ mortality is primarily due to the development of colorectal liver metastasis (CRLM). ${ }^{2-3}$ Over $50 \%$ of CRC patients will either present with synchronous disease or later develop metachronous liver metastases during their disease course. ${ }^{4}$ Despite advancements in surgical interventions and chemotherapeutics, CRLM is associated with poorer outcomes, emphasizing the need to define mechanisms and measures to monitor the spread of disease. While there are continued extensive efforts to optimize CRC screening, we are lacking an understanding of methods to identify patients at risk for development of CRLM. This is especially evident in patient populations with an alcohol use history that may correlate with advanced CRC disease and CRLM-associated mortality.

In the U.S., the five-year relative survival for patients with stage I-III CRC varies between $71-90 \%$ whereas those with metastatic liver disease have $14 \%$ survival. ${ }^{5}$ This profound decrease in survival rates demonstrates the necessity to identify patients at risk for development of metastatic disease, especially CRLM because the liver is the predominant site of CRC spread. Alcohol abuse is known to contribute to $6 \%$ of the World deaths with approximately $12 \%$ of those deaths being associated with alcohol-related carcinogenesis. Other lifestyle habits such as smoking and consumption of red meat are also associated with CRC burden.$^{6-8}$ However, alcohol consumption, in particular, has been identified as an independent risk factor of CRLM and a factor associated with poorer prognosis of CRC patients. ${ }^{8-10}$ Alcohol use correlates with increased CRLM identified at initial CRC diagnosis as well as with hepatic metastases that occur throughout the disease course. ${ }^{10-11}$ Preclinical studies have shown that the alcohol-injured liver is susceptible to developing CRLM earlier and to a greater extent compared to healthy liver. ${ }^{12-16}$

Clinical assessments have shown that CRC patients with an alcohol use history often require intensive follow-up due to reduced liver function, unresectable hepatic lesions, and poor survival rates. ${ }^{6,17-18}$ However, few reports exist concerning alcohol and CRLM risk in the U.S. indicating the need for research defining the role of alcohol consumption in CRC burden. Moreover, recent studies indicate an alarming increase in the rate of morbidity and mortality from alcohol use disorders and related diseases in the U.S. ${ }^{19}$ This is especially evident as alcohol use patterns such as heavy and episodic binge drinking may have a profound impact on disease burden and healthcare costs. As a result, there is a call for focused public health strategies to address alcohol misuse and to characterize the role of alcohol consumption as a predisposing factor to CRLM in high-risk populations.

The Midwest region of the US has one of the highest incidence rates of CRC as well as heavy and binge drinking prevalence. , $20-21^{2}$ The aberrant use of alcohol is estimated to contribute to the higher incidence of $\mathrm{CRC}$ with evidence indicating that heavy drinking increases the risk of CRC mortality. ${ }^{6}$ Additionally, the elevated risk of poor outcomes of CRC appear higher for men compared to women at similar levels of alcohol exposure. The male gender has also been established as an independent risk factor for incidence of CRLM. ${ }^{4,22-23}$ Altogether, the level of alcohol consumption prior to CRC diagnosis is considered a significant risk factor not only for cancer development but for poor outcomes as well. The geographic and sex-specific risks associated with drinking 
need to be confirmed by further research and may be benefited by the characterization of combined assessments of cancer biomarkers and alcohol consumption.

The potential of serum carcinoembryonic antigen (CEA) as a measure of advanced CRC has been demonstrated in both preclinical and clinical studies. ${ }^{11,14,24-25}$ As a result, serum CEA levels are routinely monitored in CRC patients and it is well established that elevated levels correlate with advanced disease. ${ }^{24-25}$ Importantly, CEA levels were found to be increased in $>80 \%$ of patients with distant metastasis with serial CEA measures used as a detection method for CRLM. ${ }^{24}$ However, the monitoring of CEA as an exclusive biomarker has been longdebated in that $30-40 \%$ of CRC patients at initial diagnosis do not have elevated serum CEA values. ${ }^{26}$ Moreover, it is not known if gender differences exist for serum CEA levels in relationship to advanced CRC disease. Thus, current research efforts are evaluating the role of combination measures of serum biomarkers, biochemical parameters, and lifestyle habits for better follow-up and early identification of CRC patients at high risk for the development of liver metastasis. In this single-institution retrospective study, we evaluated the associations of gender, alcohol use, and stage with CEA levels in CRC patients at the University of Nebraska Medical Center (UNMC). We hypothesized that the combination of these variables may be utilized to identify at-risk populations for CRLM in a region that has a high incidence of CRC and alcohol use disorders.

\section{Methods}

Patient Population: We performed a retrospective analysis of UNMC patients diagnosed with CRC between 2012-2019. Data were obtained from the UNMC clinical data warehouse (CDW), an i2b2-based data repository (https://www.i2b2.org/), internally referred to as CRANE (Clinical Research Analytics Environment). The i2b2 data is populated using information from the Electronic Medical Record (EMR). The inclusion criteria included CRC as the primary cancer; NAACCR stage (stages I-IV); age $>19$; and treatment at UNMC. The data extracted included gender; history of alcohol use, liver enzymes, serum CEA, and date of death or last follow-up. This study was reviewed and exempted by our Institutional Review Board.

Data Characteristics and Statistical Analysis: A population of 1,243 CRC patients in all stages (I-IV) was identified in the
CRANE database. Only twenty-seven percent of the total CRC patients had a documented alcohol use history and were included in the final analysis. Differences in CEA between subgroups of variables of interest were assessed using Kruskal-Wallis tests, and Wilcoxon Rank Sum tests for post-hoc pairwise comparisons, or Chi-Square tests when CEA was dichotomized (i.e., Low: CEA $<3.4$; High: CEA $>=3.4$ ). For modeling, initial CEA values were log transformed due to significant skewness in the data. To assess change in CEA over time, the difference in $\log$ CEA values $(\log ($ final_CEA) $-\log$ (initial_ CEA)) were divided by the length of follow up, to give the average difference in $\log$ CEA values per year. For both log of initial CEA value and average annual change in the log values of CEA as outcomes, a triple interaction between stage, gender, and alcohol use was assessed using linear regression models, which adjusted for relevant lower order interactions and main effects, as well as initial log CEA for the change model only. A significant triple interaction was further investigated by assessing gender by alcohol use interactions in separate models, one for each of the three tumor stages (I, II/III and IV). Significant two-way interactions or main effects were assessed with Bonferroni or Tukey's post-hoc adjusted pairwise comparisons, respectively. All analyses were performed using SAS software version 9.4 (SAS Institute Inc., Cary, NC).

\section{Results}

Patient Characteristics: The patient population evaluated in this study is shown in Fig. 1. A total of 1,243 patients were identified

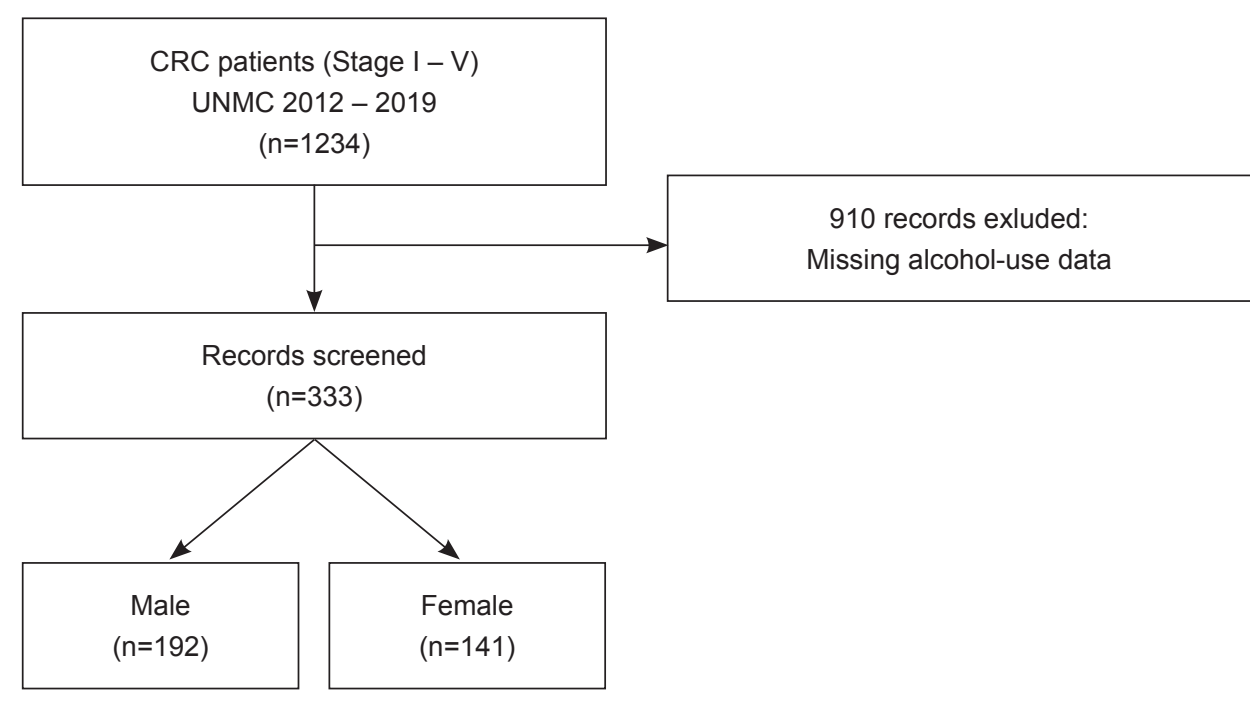

Figure 1. Flow diagram of the single-institution retrospective analysis of colorectal cancer patients with alcohol-use data. in the CRANE database as CRC patients of all stages at UNMC between the years of 2012-2019. Three hundred and thirty-three patients $(26.7 \%)$ had information on alcohol use (dichotomized as either no or some use) that was recorded in the EMR. This cohort evaluated was comprised of 192 male and 141 female subjects. Alcohol use did not significantly differ across the tumor stages, as shown in Table 1. However, significantly fewer females reported alcohol use $(27.7 \%)$ compared to males $(44.3 \%), p=0.002$.

\section{Analysis of Alcohol Use and Serum CEA} levels: A linear regression analysis of the $\log$ of serum CEA levels recorded near or at the date of CRC diagnosis (initial CEA) is represented in Fig. 2. The triple interaction between stage, gender, and alcohol use was not significant $(\mathrm{p}=0.84)$. However, the main effect of stage was significant, where initial serum CEA values (i.e., at time of CRC diagnosis) were found to be significantly increased in Stage IV compared to the Stages I $(\mathrm{p}<0.0001)$ and Stages II/III $(\mathrm{p}<0.0001)$, as expected.

The utility of CEA measures as a prognostic indicator of advanced disease and likely CRLM is further emphasized by the analysis of mortality and the initial recorded measure of CEA in the patient cohort (Fig. 3). The data indicate that elevated measures of initial serum CEA levels are associated with increased all-cause mortality $(33.0 \%$ for CEA $>3.4 \mathrm{ng} / \mathrm{ml}$ vs $10.4 \%$ for CEA $<3.4 \mathrm{ng} / \mathrm{ml}, \mathrm{p}<$ 0.0001). Finally, an assessment of the yearly change in log serum CEA values over time demonstrated a significant triple interaction between gender, stage, and alcohol use $(\mathrm{p}=0.01)$. To further assess this relationship,

\section{Missing alcohol-use data}


the gender by alcohol two-way interaction was assessed in separate models, one for each stage. This interaction between gender and alcohol use was only significant in the Stage IV patient model $(\mathrm{p}=0.02)$. While there was no significant difference in the rate of change in log CEA between the two alcohol use groups among Stage IV women $(p=0.64)$, men in this stage who used alcohol had a significantly higher rate of change in log CEA than men who did not $(\mathrm{p}=0.02)$ (Fig. 4).

\section{Discussion}

Alcohol intake is a well-established risk factor for primary CRC. ${ }^{27-28}$ Alcohol consumption has also been shown to be an independent risk factor of CRLM and poorer prognosis of CRC patients. ${ }^{9-10,18}$ The current literature supports that alcohol creates a hepatic microenvironment susceptible to tumor cell seeding and growth. ${ }^{13-15,29}$ However, few reports exist concerning alcohol and CRLM risk in the U.S. Specifically, there is a paucity of data in Midwest states such as Nebraska that report high rates of alcohol use disorders and risk of colorectal cancers ${ }^{1,20-21}$ which prompted this retrospective analysis of alcohol use in CRC patients treated at the University of Nebraska Medical Center.

Recent studies indicate an alarming increased rate of morbidity and mortality from alcohol use disorders in the U.S. with the call for focused public health strategies to address this serious situation. ${ }^{6,18-19}$ The National Institute on Alcohol Abuse and Alcoholism (NIAAA) has recommended that patients should be

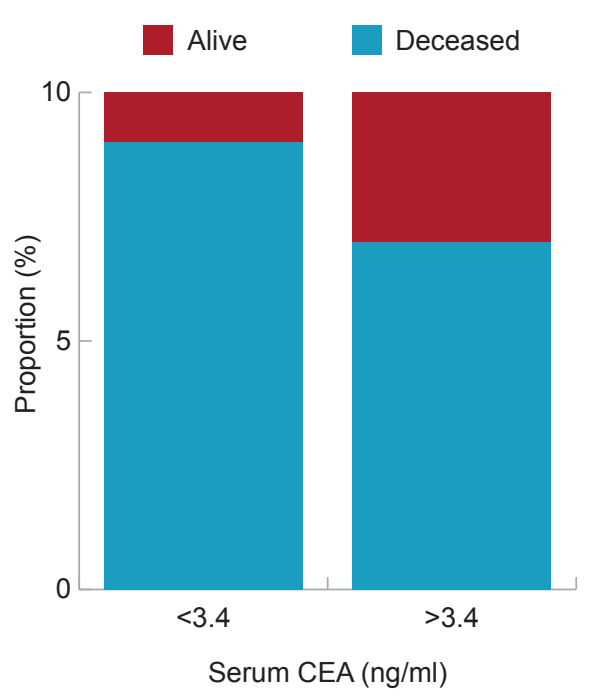

Figure 3. CRC mortality in relationship to initial serum CEA values. The percentage of CRC patients (alive or deceased) compared to the serum CEA levels obtained at initial visit following colorectal cancer diagnosis.
Table 1.

Patient Characteristics, Alcohol use

\begin{tabular}{lllll} 
& $(\mathbf{n})$ & $\begin{array}{l}\text { Alcohol use } \\
\text { (some) }\end{array}$ & $\begin{array}{l}\text { Alcohol use } \\
\text { (none) }\end{array}$ & P-value \\
\hline Total CRC patients with alcohol use documented & 333 & 124 & 209 & \\
\hline Sex (\% of total) & & & & 0.002 \\
$\quad$ Male & 192 & $85(44.3 \%)$ & $107(55.7 \%)$ & \\
$\quad$ Female & 141 & $39(27.7 \%)$ & $102(72.3 \%)$ & \\
\hline Stage (\% of total, 13 missing) & & & & 0.28 \\
I & 119 & $50(42.0 \%)$ & $69(58.0 \%)$ & \\
II/III & 97 & $36(37.1 \%)$ & $61(62.9 \%)$ & \\
IV & 104 & $33(31.7 \%)$ & $71(68.3 \%)$ & \\
\hline
\end{tabular}

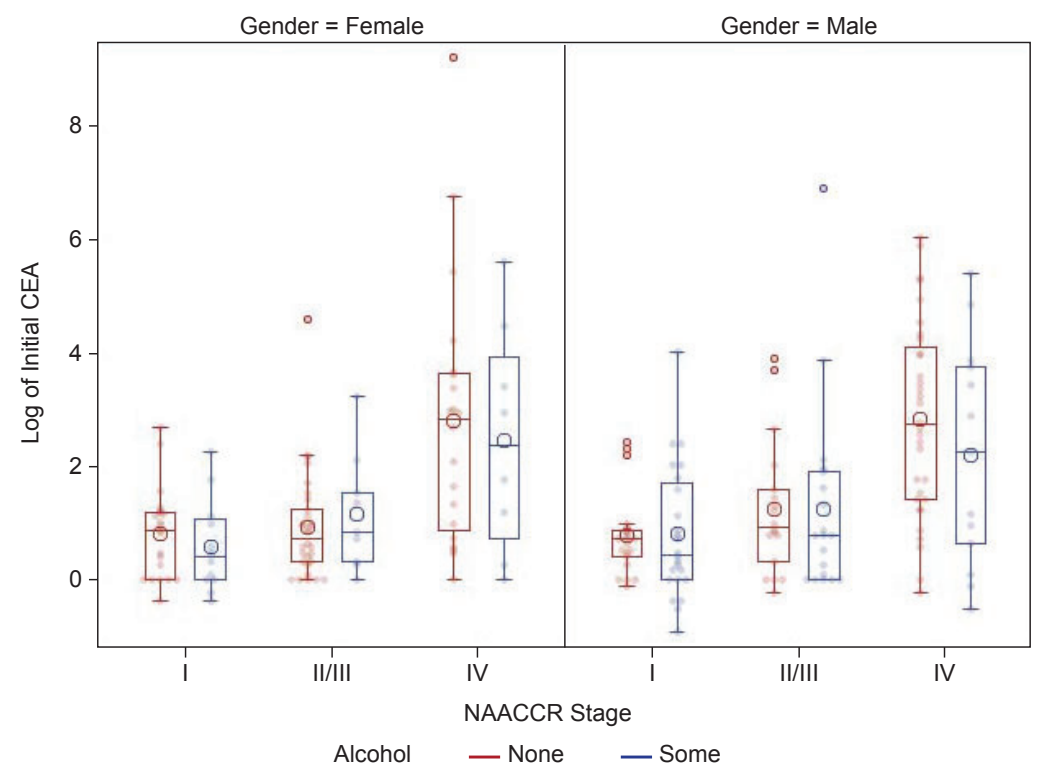

Figure 2. Triple interaction between stage, gender, alcohol use (none vs some) and initial CEA values recorded in colorectal cancer patients. Stage IV had significantly higher log initial CEA values than the other two stage groups $(p<0.0001)$. NAACCR, North American Association of Central Cancer Registries Stages I, II/III and IV.

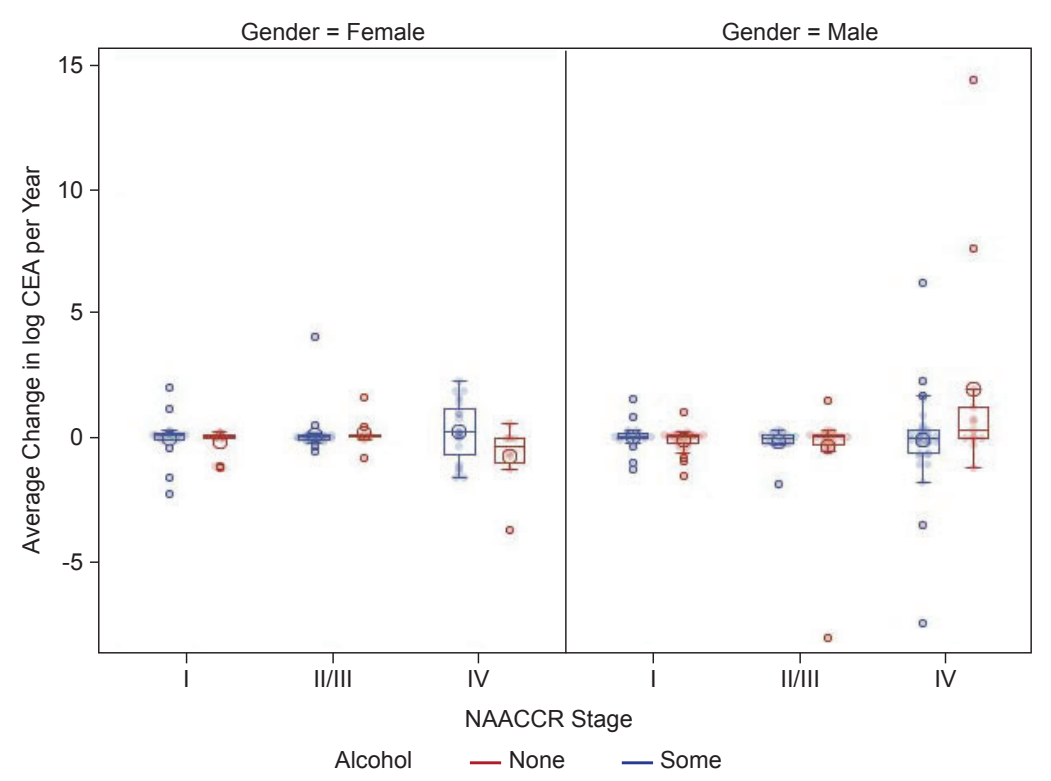

Figure 4. Average change in log CEA per year stratified by gender and stage (NAACCR, North American Association of Central Cancer Registries Stages I, II/III and IV). A significant interaction was observed between gender and alcohol use in Stage IV male patients $(p=0.02)$. 
questioned about the pattern of alcohol consumption in addition to the number of drinks consumed.$^{30}$ At UNMC, the Electronic Medical Record (EMR) alcohol history includes frequency of alcohol use, drinks per occasion, and type of alcohol (wine, beer, liquor). However, questions are lacking for the presence of binge episodes and prior consumption patterns. Further, a review of the EMR data at our institution indicates that alcohol use data is not recorded in discrete data fields for the majority of patients. While some EMR records contain physician notes concerning alcohol consumption, this data is inconsistent and variable in content. In agreement with this review, the current retrospective analysis determined that only $27 \%$ of patient records identified for inclusion were found to have alcohol use information in the i2B2 repository. Additionally, the majority of records reporting alcohol use were not complete in relation to number of drinks and type of alcohol consumed. As a result, this study was only able to report alcohol use as none or some alcohol consumed. This limitation associated with poor documentation of alcohol use at UNMC is also observed as a widespread problem seen in across institutions worldwide; several analyses have demonstrated that alcohol use history and diagnoses of alcohol use disorder are greatly under documented in the EMR..$^{31-33}$ This is a major concern as alcohol use data could prove to be meaningful in the investigations of several disease processes, including colorectal cancer.

Despite the limitation of missing alcohol use data, the relationship of alcohol use history, CRC stage, and tumor marker measures was assessed in a cohort of 333 CRC patients. The tumor marker studied was CEA, a glycoprotein of the immunoglobulin gene family that codes for several tumorigenic adhesion proteins.${ }^{34} \mathrm{CEA}$ serum measures have proven to be a useful marker for CRC with a significant benefit found for the assessment of prognosis and liver metastasis. ${ }^{35}$ Studies indicate that patients with high preoperative CEA serum concentrations ( $>5 \mathrm{ng} / \mathrm{ml}$ ) have worse outcomes as well as disease recurrence. ${ }^{35-37}$ Importantly, CEA levels are significantly elevated in patients with distant metastasis and the measure of serial CEA levels over time is used as an accurate detection method for CRLM. ${ }^{17,24}$ The results of our retrospective analysis are consistent with reports for the use of CEA as a marker for advanced disease. ${ }^{26,38} \mathrm{CEA}$ levels increased significantly with tumor stage and mortality correlated with elevated serum CEA levels. However, the clinical significance has not been defined for the monitoring of initial CEA values compared to serial CEA measures in relationship to behavioral and environmental factors such as alcohol use. Furthermore, it is unclear if CEA levels differ between men and women and what role a history of alcohol consumption might play. Our data indicates that initial, likely preoperative, CEA values are significantly higher when comparing patients in stage IV to stage I. This is consistent with other studies and the well-defined role of CEA in predicting advanced disease stage (i.e. CRLM) with minimal increases in CEA levels seen after initial diagnosis in early disease stages (i.e. stage I). ${ }^{24,26,38}$ Further, in this study with limited alcohol use data, we were not able to show an association between alcohol use and initial CEA serum values during any stage of $\mathrm{CRC}$ disease. Future analyses are needed to determine if such a relationship exists.

With respect to gender differences, this study indicates a potential role of alcohol use and serial CEA values in males with advanced CRC disease. This is clinically relevant as the incidence and mortality of colorectal cancer is known to be higher in males. ${ }^{1,39}$ Several retrospective, populationbased studies have also found male gender to be a risk factor for both synchronous and metachronous liver metastases. ${ }^{4,22-23}$ However, there are no gender-specific mechanisms for CRC diagnosis or monitoring based on current society guidelines. This includes initial screening for CRC and surveillance protocols in patients with stage II and III disease following resection..$^{35,40}$ The present analysis found that male alcohol users with stage IV disease showed a greater increase in serial CEA levels than males who did not use alcohol, a pattern that was not seen in stage IV females. The current literature supports that male sex, alcohol use, and elevated CEA levels are each independent risk factors for the presence of metastatic liver disease. Thus, males with a history of alcohol use should be recognized as a high-risk population for metastatic involvement and serial CEA monitoring may have increased utility in these patients.

In summary, in this study alcohol use was associated with increased average annual change in CEA among stage IV males, at least in the small subset of patients where alcohol use was documented. Considering the growing burden of alcohol related disease, further investigation regarding the implications of alcohol use on development of CRLM may be clinically meaningful and demonstrate the importance of obtaining alcohol use data in CRC patients. Given the substantial mortality associated with CRLM, research efforts are needed to focus on identifying vulnerable patients. A detailed alcohol use history should be obtained in all patients with CRC as it has prognostic value. Our retrospective analysis was limited by missing alcohol use data for a majority of patients treated for CRC. A prospective study is warranted for a better understanding and definitive conclusions with regards to the implications of alcohol use on liver metastases in CRC.

Author Contributions: Conceptualization, LMK, AWP, BLM; Methodology, LMK, AWP, WSC, KKS, BLM; Data Analysis, LMK, AWP, WSC, KKS, AM, MT, BLM; Original Draft Preparation, LMK, AWP, BLM; Manuscript Review and Editing, LMK, AWP, WSC, KKS, AM, MT, BLM. All authors have read and agreed to the published version of the manuscript.

\section{References}

1 Siegel RL, Miller KD, Goding Sauer A, et al., Colorectal cancer statistics, 2020. CA Cancer J Clin 2020; 70: 145-164.

2 Anaya DA, Becker NS, Richardson P, et al., Use of administrative data to identify colorectal liver metastasis. J Surg Res 2012; 176: 141-6.

3 Paschos KA, Majeed AW, Bird NC. Natural history of hepatic metastases from colorectal cancer-pathobiological pathways with clinical significance. World J Gastroenterol 2014; 20: 3719-37.

4 Engstrand J, Nilsson H, Stromberg C, et al., Colorectal cancer liver metastases - a population-based study on incidence, management and survival. BMC Cancer 2018; $18: 78$.

5 Siegel RL, Miller KD, Jemal A. Cancer statistics, 2020. CA Cancer J Clin 2020; 70: 7-30.

6 Cai S, Li Y, Ding Y, et al., Alcohol drinking and the risk of colorectal cancer death: a meta-analysis. Eur $J$ Cancer Prev 2014; 23: 532-9.

7 Carr PR, Jansen L, Walter V, et al., Associations of red and processed meat with survival after colorectal cancer and differences according to timing of dietary assessment. Am J Clin Nutr 2016; 103: 192-200.

8 Haggar FA, Boushey RP. Colorectal cancer epidemiology: incidence, mortality, survival, and risk factors. Clin Colon Rectal Surg 2009; 22: 191-7.

9 Haddad AJ, Bani Hani M, Pawlik,TM, et al., Colorectal liver metastases. Int J Surg Oncol 2011; 2011: 285840.

10 Maeda M, Nagawa H, Maeda T, et al., Alcohol consumption enhances liver metastasis in colorectal carcinoma patients. Cancer 1998; 83: 1483-8.

11 Dong H, Tang J, Li LH, et al., Serum carbohydrate antigen 19-9 as an indicator of liver metastasis in colorectal carcinoma cases. Asian Pac J Cancer Prev 2013; 14: 909-13.

12 Cernigliaro C, D'Anneo A, Carlisi D, et al., Ethanolmediated stress promotes autophagic survival and aggressiveness of colon cancer cells via activation of Nrf2/HO-1 pathway. Cancers 2019; 11: 505.

13 Im HJ, Kim HG, Lee JS, et al., A preclinical model of chronic alcohol consumption reveals increased metastatic seeding of colon cancer cells in the liver. Cancer Research 2016; 76: 1698-1704.

14 Mohr, A. M, Gould, J. J, Kubik, J. L., et al., Enhanced colorectal cancer metastases in the alcohol-injured liver. Clinical \& Experimental Metastasis 2017; 34 : 171-184. 
15 Xu M, Wang S, Qi Y, et al., Role of MCP-1 in alcoholinduced aggressiveness of colorectal cancer cells. $\mathrm{Mol}$ Carcinog 2016; 55: 1002-11.

16 Zheng K, Yu J, Chen Z, et al., Ethanol promotes alcohol-related colorectal cancer metastasis via the TGF- $\beta /$ RUNX3/Snail axis by inducing TGF- $\beta 1$ upregulation and RUNX3 cytoplasmic mislocalization. EBioMedicine 2019; 50: 224-237.

17 Bhattacharjya S, Aggarwal R, Davidson BR. Intensive follow-up after liver resection for colorectal liver metastases: results of combined serial tumour marker estimations and computed tomography of the chest and abdomen - a prospective study. Br J Cancer 2006; 95: 21-6.

18 Walter V, Jansen L, Ulrich A, et al., Alcohol consumption and survival of colorectal cancer patients: a population-based study from Germany. Am J Clin Nutr 2016; 103: 1497-506.

19 Bataller R, Arteel GE, Moreno C, et al., Alcoholrelated liver disease: Time for action. J Hepatol 2019; 70: 221-222.

20 Kanny D, Naimi TS, Liu Y, et al., Annual total binge drinks consumed by U.S. adults, 2015. American journal of preventive medicine 2018; 54: 486-496.

21 Services D.o.H.a.H. Colorectal (Colon \& Rectum) Cancer in Nebraska; 2018.

22 Gaitanidis A, Machairas N, Alevizakos M, et al., Predictive Nomograms for Synchronous Liver and Lung Metastasis in Colon Cancer. J Gastrointest Cancer 2020; 51: 925-931.

23 Manfredi S, Lepage C, Hatem C, et al., Epidemiology and management of liver metastases from colorectal cancer. Ann Surg 2006; 244: 254-9.

24 Pakdel A, Malekzadeh M, Naghibalhossaini F. The association between preoperative serum CEA concentrations and synchronous liver metastasis in colorectal cancer patients. Cancer Biomark 2016; 16 : 245-52.

25 Stojkovic Lalosevic M, Stankovic S, Stojkovic M, et al., Can preoperative CEA and CA19-9 serum concentrations suggest metastatic disease in colorectal cancer patients? Hell J Nucl Med 2017; 20: 41-45.

26 Kahi CJ, Anderson JC, Rex DK. Screening and surveillance for colorectal cancer: state of the art. Gastrointest Endosc 2013; 77: 335-50.

27 Nelson DE, Jarman DW, Rehm J, et al., Alcoholattributable cancer deaths and years of potential life lost in the U.S. Am J Public Health 2013; 103: 641-8.

28 Wang Y, Duan H, Yang H, et al., A pooled analysis of alcohol intake and colorectal cancer. International Journal of Clinical and Experimental Medicine 2015; 8: 6878.

29 McVicker B, Tuma DJ, Lazure KE, et al., Alcohol, carcinoembryonic antigen processing and colorectal liver metastases. Adv Exp Med Biol 2015; 815: 295 311.

30 Allen JP, Wilson VB. Assessing alcohol problems: A guide for clinicians and researchers. U.S. Department of Health and Human Services, Public Health Service, National ... : 2003

31 Abidi L, Oenema A, van den Akker M, et al., Do general practitioners record alcohol abuse in the electronic medical records? A comparison of survey and medical record data. Current Medical Research and Opinion 2018; 34: 567-572.

32 Hallgren KA, Witwer E, West I, et al., Prevalence of documented alcohol and opioid use disorder diagnoses and treatments in a regional primary care practicebased research network. Journal of Substance Abuse Treatment 2020; 110: 18-27.

33 Mitchell AJ, Meader N, Bird V, et al., Clinical recognition and recording of alcohol disorders by clinicians in primary and secondary care: metaanalysis. The British Journal of Psychiatry 2012; 201: 93-100.
34 Blumenthal RD, Leon E, Hansen HJ, et al., Expression patterns of CEACAM5 and CEACAM6 in primary and metastatic cancers. BMC Cancer 2007; 7: 2 .

35 Beauchemin N, Arabzadeh A. Carcinoembryonic antigen-related cell adhesion molecules (CEACAMs) in cancer progression and metastasis. Cancer Metastasis Rev 2013; 32: 643-71.

36 Giuliante F, Panettieri E, Ardito F, et al., Circulating tumor cell-related transcripts in blood as prognostic biomarkers of early recurrence after liver resection for colorectal metastases. Int J Biol Markers 2019; 34: 269-275.

37 Hostetter RB, Augustus LB, Mankarious R, et al., Carcinoembryonic antigen as a selective enhancer of colorectal cancer metastasis. J Natl Cancer Inst 1990; 82: $380-5$.

38 Hanke B, Riedel C, Lampert S, et al., CEA and CA 19-9 measurement as a monitoring parameter in metastatic colorectal cancer (CRC) under palliative first-line chemotherapy with weekly 24 -hour infusion of high-dose 5-fluorouracil (5-FU) and folinic acid (FA). Ann Oncol 2001; 12: 221-6.

39 Park SM, Lim MK, Shin SA, et al., Impact of prediagnosis smoking, alcohol, obesity, and insulin resistance on survival in male cancer patients: National Health Insurance Corporation Study. Journal of Clinical Oncology 2006; 24: 5017-5024.

40 Rex DK, Boland CR, Dominitz JA, et al., Colorectal cancer screening: Recommendations for physicians and patients from the U.S. Multi-Society Task Force on Colorectal Cancer. Gastrointest Endosc 2017; 86: $18-33$. 